\title{
Improvements in Transport Phenomena Teaching
}

\author{
Pierre Proulx ${ }^{l}$, Francis B. Lavoie ${ }^{l}$ \\ ${ }^{1}$ Département de génie chimique et de génie biotechnologique, Université de Sherbrooke \\ Pierre.Proulx@USherbrooke.ca
}

\begin{abstract}
Over the last years, the Department of Chemical Engineering of the Universite de Sherbrooke has made a major change: the Matlab teaching has been replaced by Python. This programming language shift allowed the access to multiple powerful tools which were not available before. The Transport Phenomena courses involve theoretical understanding, the use of multiple physicochemical properties as well as complex mathematical equation solving. Opensource Python packages were then substantially implemented in these courses and all exercises and examinations are now performed with computers. Moreover, the Transport Phenomena courses are now given in flipped classroom with the use of a novel web platform developed in our Department. This platform allows to execute Python codes directly on the website and allows to follow and to quantify the overall progress of the students in the course.
\end{abstract}

Keywords: Transport phenomena; Python; Jupyter Notebooks; Sympy; Flipped classroom;

\section{INTRODUCTION}

Modern high-level programming languages simplifies the use of mathematical tools to resolve engineering problems. Jupyter Notebooks are now considered as both a powerful teaching support and a great problem resolution tool [1]. The most popular symbolic Python module, Sympy, is now considered as equivalent to other commercial ones such as the Symbolic Toolbox in Matlab [2].

Transport Phenomena is a second-year course where the symbolic manipulation tools included in the Matlab package have been used for years to deal with the rather intense mathematical content. Students taking up the Transport Phenomena courses of the Department of Chemical and Biotechnological Engineering of the Université de Sherbrooke having been introduced to Python in their first-year courses, it was natural to switch to the use of Sympy, and further to the use of Jupyter Notebooks.
We took this opportunity to also change how the course is taught. The flipped classroom being an increasingly widespread teaching method, principally thanks to new and constantly improved IT academic tools [3], we also opted for this blended learning model. We also developed our own web platform, called Catalyseur.ca, to better incorporate learning contents and problem solving with modern and open-source Python tools.

\section{THE PYTHON SHIFT}

The Sympy package [4] is a modern tool to solve linear equations as well as to resolve derivatives, integrals and differential equations. This package is also introduced during the first-year mathematical course and is presented as a tool to solve complex mathematical problems, as the students will face more concretely in their following study year during the Transport Phenomena courses.

The Python shift also allowed access to multiple opensource packages related to engineering. For example, the students no longer use tables of properties and empirical equations to complete the exercises and examinations. They rather use the CoolProp [5] and Thermo [6] packages to retrieve physicochemical properties or the Fluid [7] package when working with fluid mechanisms and dimensionless numbers. The use of such powerful packages and their implementation into Python codes is introduced in the first-year courses. The students are then already well equipped to write Python codes with these packages and can focus on the theoretical comprehension when assisting to the Transport Phenomena courses.

The nature of the principles leading to the equations and their associated boundary conditions remain the focus. The example shown in Figure 1 displays a rather complex example from Bird's Transport Phenomena where a model for a catalytic reactor model leads to the simultaneous solution of three second order ODEs. The solution of such problem involves a very high level of algebraic manipulations that are greatly simplified using Sympy in a Notebook. In fact, a better, more physically realistic solution to the problem is obtained readily and enables the students to explore and see the 
influence of the various parameters and properties of the reactor.

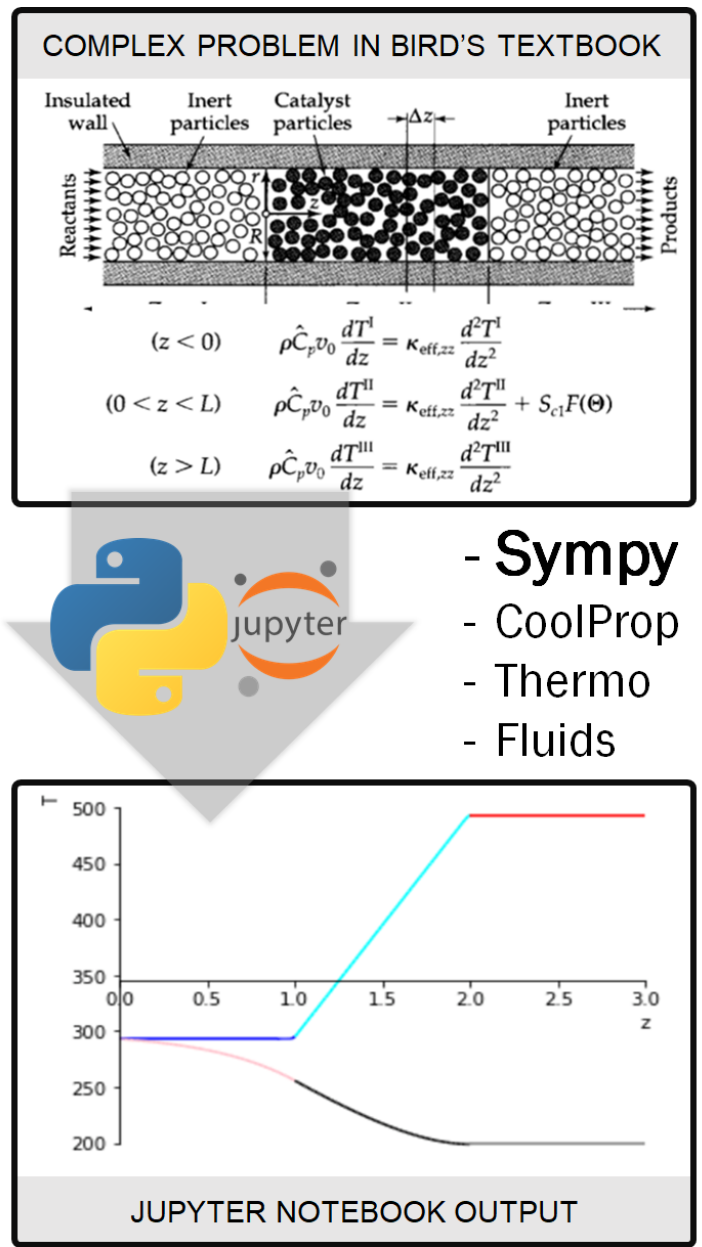

Fig. 1. From complex problem solving in Bird's text book to Jupyter Notebooks. The use of Python also allows the use of open-source powerful packages:

Sympy for mathematical equation solving and CoolProp, Thermo and Fluids to get physicochemical properties.

\section{JUPYTER NOTEBOOKS}

The use of Jupyter Notebooks was an important component of the inverted class method as it easily combines audio, video and written material in an interactive mathematical manipulation environment.

The use of Jupyter Notebooks by the engineering community skyrocketed over the last years [8]. This Python code format allows to alternatively present codes and their output on a single web page (Figure 2).
The outputs can either be in the form of a simple variable value display, a text, or in the form of a graph or a Latex equation. As the outcomes of Transport Phenomena problems are often in the form of graphs or equations, the use of Jupyter Notebooks is especially convenient for these courses. The Python code can directly be edited on a Notebook web page and the output is automatically updated after a modification. Moreover, a Jupyter Notebook is based on a single file, even in the presence of figures in the code outputs. A Jupyter Notebook can then easily be shared to the students, and vice versa. Indeed, exercises and reports can be written with this versatile tool as it allows to mix Python codes, texts and graphs.

Their simplicity led us to choose Jupyter Notebooks as a medium for teaching theoretical contents and problem-solving examples in the context of inverted classes as well as the medium to solve exercises and examinations. Computer examinations often lead to issues, especially when dealing with multiple files. The use of Jupyter Notebooks with their single file characteristic minimises the risks of issues. Indeed, we observed considerably less problems with computer examinations by using Jupyter Notebooks compared to examinations relying on the completion of Matlab or regular Python scripts.

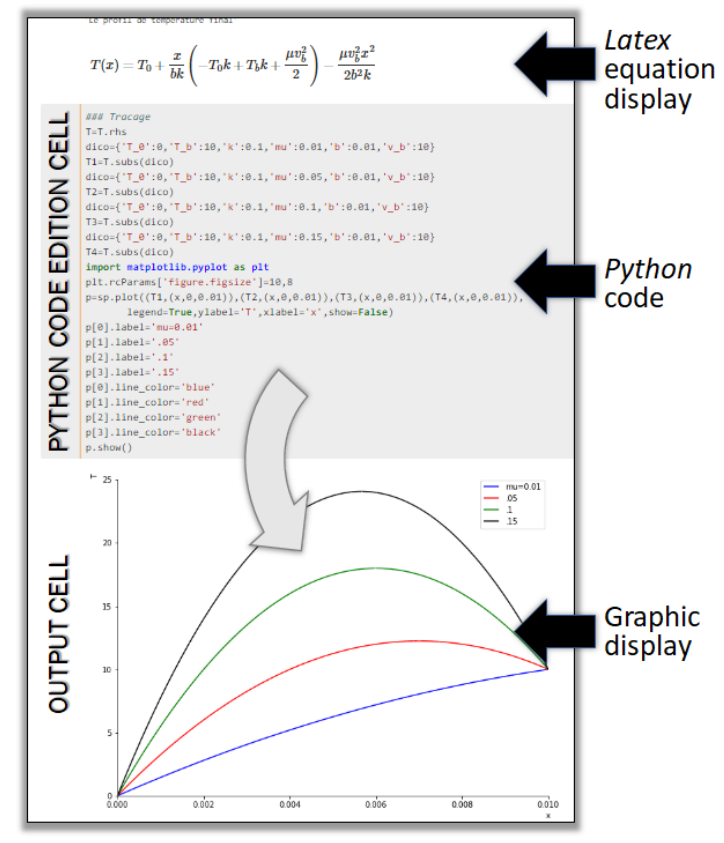

Fig. 2. Regular Jupyter Notebook. Python code cells and output cells are alternatively presented in one single web page. Each code cell can be directly edited on the web page, which then automatically updates the following output. 


\section{THE WEB PLATFORM}

The Transport Phenomena courses also use our new Catalyseur.ca platform developed in our department. The Jupyter Notebooks are integrated into the website besides the creation of videos specifically related to Transport Phenomena notions.

Original exercises were also written and integrated to the web platform. Each exercise has its own web page. Students can edit their Python codes and execute them directly on each exercise page as if they were working in a Jupyter Notebook. There is no difference between the output obtained from a Python code executed from the regular Jupyter Notebook interface and the same code executed from the Catalyseur.ca web platform. The students can work with the web platform to complete the exercises and then answering the examination questions with the Jupyter Notebook regular interface when the Internet access is blocked.

Moreover, all Python codes written by the students are saved in a database and these codes are all accessible by the teacher. In addition, the navigation of the students through Catalyseur.ca generates data that are then used to automatically generate statistics relative to the completion of exercises and the understanding of notions. The teacher can easily know which subjects and exercises are less understood and can also access to non-completed codes. The teacher can then easily identify what are the problems encountered by the students while solving exercises. If necessary, the teacher can make the point on a topic with the entire class.

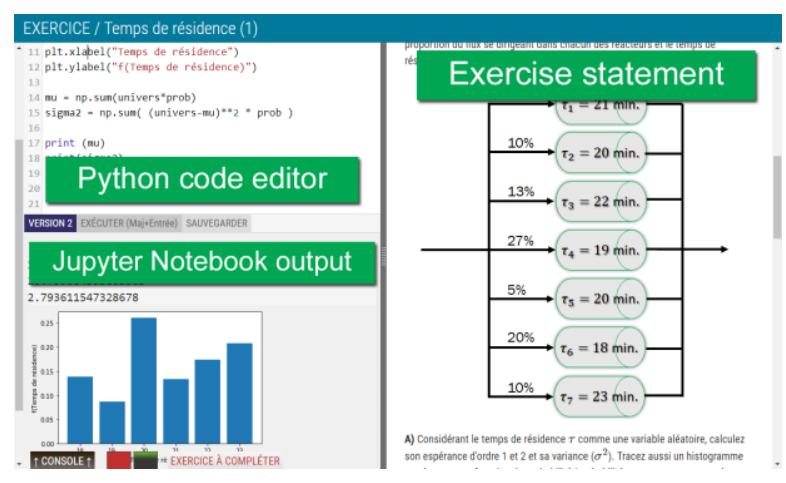

Fig. 3. Catalyseur.ca exercise page. The Python code editor, the Jupyter Notebook output, the exercise statement as well as forums allowing the students to ask questions with each other are all integrated into one single web page.
We have questioned many students about the web platform. They appreciate the video courses in French which can be replayed and stopped any time. This point was especially raised by students having learning disabilities. The students also appreciate the simplicity of the interface, compared to common learning management systems such as Moodle. They also appreciate to complete the exercises directly in a web browser, without having to install anything on their personal computer.

\section{CONCLUSION}

With the updated second-year Transport Phenomena courses adapted to modern computer tools, we can alleviate the burden of the mathematical juggling and put the emphasis on the physical phenomena.

This update would have not been possible without the open-source shift and the major changes in first-year courses. The development of a novel web platform also considerably improved student learning assessments.

\section{References}

[1] Roehl, A., Reddy, S. L., \& Shannon, G. J. (2013). The flipped classroom: An opportunity to engage millennial students through active learning. Journal of Family and Consumer Sciences, 105(2), 44.

[2] Shen, H. (2014). Interactive notebooks: Sharing the code. Nature, 515(7525), 151.

[3] Millman, K. J., \& Aivazis, M. (2011). Python for scientists and engineers. Computing in Science \& Engineering, 13(2), 9-12

[4] Meurer, A., Smith, C. P., Paprocki, M., Čertík, O., Kirpichev, S. B., Rocklin, M., ... \& Rathnayake, T. (2017). SymPy: symbolic computing in Python. PeerJ Computer Science, 3, e103.

[5] Bell, I. H., Wronski, J., Quoilin, S., \& Lemort, V. (2014). Pure and pseudo-pure fluid thermophysical property evaluation and the open-source thermophysical property library CoolProp. Industrial \& engineering chemistry research, 53(6), 2498-2508.

[6] Bell, C. (2016). thermo: Chemical properties component of Chemical Engineering Design Library (ChEDL), https://github.com/CalebBell/thermo 
Proc. 2018 Canadian Engineering Education Association (CEEA-ACEG18) Conf.

[7] Bell, C. (2016). fluids: Fluid dynamics component of Chemical Engineering Design Library (ChEDL), $\underline{\text { https://github.com/CalebBell/fluids }}$

[8] Kluyver, T., Ragan-Kelley, B., Pérez, F., Granger, B. E., Bussonnier, M., Frederic, J., ... \& Ivanov, P. (2016, May). Jupyter Notebooks-a publishing format for reproducible computational workflows. In ELPUB (pp. 87-90).

CEEA18; Paper 056 